\title{
Computation of Normal Depth in Parabolic Cross Sections Using The Rough Model Method
}

\author{
Bachir ACHOUR
}

Research Laboratory in Subterranean and Surface Hydraulics (LARHYSS) University of Biskra, PO Box 145 RP 07000 Biskra, Algeria

\begin{abstract}
A new method is applied to calculate the normal depth in an open channel of parabolic cross section. This is the rough model method whose main particularity is to ignore the flow resistance coefficients, such as Chezy's coefficient and manning's roughness coefficient. The method is applied to a referential rough model, whose friction coefficient is constant, which explicitly express the hydraulic and geometric characteristics of the model such as aspect ratio. By means of a non-dimensional correction factor, the normal depth is explicitly deduced. The rough model method is applicable to the entire domain of turbulent flow.
\end{abstract}

Keywords: Discharge, Energy slope, Normal depth, Parabolic channel, Rough model method, Turbulent flow.

\section{INTRODUCTION}

Normal depth plays a significant role in the design of open channels and in the analysis of the non-uniform flow as well. Searching for earlier literature, one can find some methods of uniform flow computation. For the most part, the well known Chezy and Manning resistance equation are extensively used. Due to their implicit form, graphical methods have been presented in the past for uniform flow computation in the common rectangular, trapezoidal, triangular and circular cross sections [1-3]. For these, explicit solutions for normal depth have been proposed afterwards [4, 5]. The most relevant study is certainly that of Swamee and Rathie [6], in which exact analytical equations for normal depth have been reported for rectangular, trapezoidal and circular cross sections. However, the solution is given in terms of an unlimited series whose application to the use of the engineer is not at all handy. For round-bottomed triangular, round-cornered rectangular and parabolic cross sections, exact or approximate solutions are not yet available. For these sections, graphical methods have been proposed by Babaeyan-Koopaei [7] for parabola of second degree, using the Manning's resistance equation. For parabolic cross section, Achour and Khattaoui [8] derived three explicit relations for the aspect ratio, depending on the value of the relative conductivity. The proposed relationships are valid in a wide practical range of relative conductivity. In recent years, graphical methods were replaced by iterative methods for all known geometric profiles of channels [9, 10]. In current methods of calculation, the major problem

*Address correspondence to this author at the Research Laboratory in Subterranean and Surface Hydraulics, Biskra University, PO Box 145 RP 07000 Biskra Algeria; Tel: 00213557263947; Fax: 0021333742481;

E-mail: bachir.achour@larhyss.net lies not in their iterative nature but rather in the fact that they consider the coefficient of Manning or Chezy as a given data of the problem. It is here that lies the real difficulty because the question is how to impose these coefficients as they depend on the normal depth sought. Even with a lot of experience, it is very difficult or impossible to set the value of these coefficients in advance, before calculating the normal depth. The only practical measurable parameter which is related to the internal state of the channel wall is the absolute roughness, usually referred to $\varepsilon$. It is this parameter that must be, in principle, a given data of the problem instead of Chezy and Manning coefficients. Currently, there is no explicit method that considers this parameter. It is in this context that this study is proposed, based on a new method known as the Rough Model Method (RMM) [11-18]. This method does not require Manning's roughness coefficient or Chezy's coefficient. For the calculation of normal depth in parabolic open channel, it requires only measurable parameters in practice, namely the discharge $Q$, the longitudinal slope $i$, the geometric elements of the channel, the absolute roughness $\varepsilon$ and the kinematic viscosity $v$ of the flowing liquid. The method is based on geometric and hydraulic characteristics of a referential rough model whose parameters are well defined. With a non-dimensional correction factor of linear dimension, these parameters are used to derive those of the studied channel, especially normal depth. In the RMM, there is no restriction in the involved parameters and the resulting equations are valid in the entire domain of turbulent flow, corresponding to Reynolds number $R \geq 2300$ and relative roughness $\varepsilon / D_{h}$ varying in the wide rang $[0 ; 0.05]$. A calculation example is presented to better understand the calculation procedure and to appreciate its simplicity and efficiency. 


\section{BASIC EQUATIONS}

The study is based on the three turbulent flow equations, namely Darcy-Weisbach equation [19], Colebrook-White equation [20] and Reynolds number formula. The DarcyWeisbach equation gives the longitudinal slope of a conduit or channel as follows:

$i=\frac{f}{D_{h}} \frac{Q^{2}}{2 g A^{2}}$

where $Q$ is the discharge, $g$ is the acceleration due to gravity, $A$ is the wetted area, $D_{h}$ is the hydraulic diameter and $f$ is the friction factor given by the well known Colebrook-White formula as:

$$
\frac{1}{\sqrt{f}}=-2 \log \left(\frac{\varepsilon / D_{h}}{3.7}+\frac{2.51}{R \sqrt{f}}\right)
$$

where $\varepsilon$ is the absolute roughness and $R$ is the Reynolds number which can be expressed as :

$R=\frac{4 Q}{P v}$

where $v$ is the kinematic viscosity and $P$ is the wetted perimeter.

\section{REFERENTIAL PARABOLIC ROUGH MODEL}

All geometric and hydraulic characteristics of the rough model are distinguished by the symbol $"{ }^{-} "$. Fig. (1) compares the geometric and hydraulic characteristics of the current channel with those of its rough model. The rough model is particularly characterized by $\bar{\varepsilon} / \overline{D_{h}}=0.037$ as the arbitrarily assigned relative roughness value, where $\overline{D_{h}}$ is the hydraulic diameter. The chosen relative roughness value is so large that the prevailed flow regime is fully rough. Thus, the friction factor is $\bar{f}=1 / 16$ according to Eq. 2 for $R=\bar{R}$ tending to infinitely large value. The rough model is also characterized by the top width $\overline{T_{m}}=T_{m}$, the height $\overline{Y_{m}}=Y_{m}$ and the longitudinal slope $\bar{i}=i$ (Fig. 1). The discharge is $\bar{Q}=Q$ implying that the normal depth $\bar{y}_{n}$ is such that $\overline{y_{n}} \neq y_{n}$ and even $\overline{y_{n}}>y_{n}$.

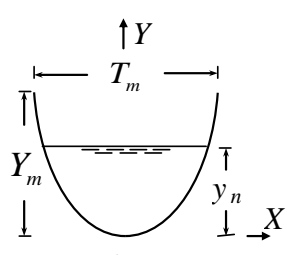

a)

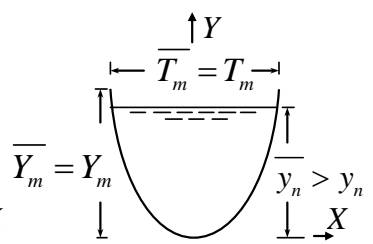

b)
Fig. (1). Schematic representation of normal depth in parabolic cross section.

a) Current channel. b) Rough model

It has been demonstrated in an earlier study [8] that the aspect ratio $\zeta_{n}$ of the water area, known also as the nondimensional normal depth, is expressed for a parabola of second degree as: $\zeta_{n}=\sqrt{y_{n} / B}$

where:

$B=T_{m}^{2} / Y_{m}$

$B$ is a known parameter since the geometric elements $T_{m}$ and $Y_{m}$ are given in practice. Considering the fact that $\overline{T_{m}}=T_{m}$ and $\overline{Y_{m}}=Y_{m}$, one can write for the rough model $\bar{B}=B$ according to Eq. (5). Applying Eq. (1) to the rough model leads to:

$i=\frac{\bar{f}}{\overline{D_{h}}} \frac{Q^{2}}{2 g A^{-2}}$

Bearing in mind that $\overline{D_{h}}=4 \bar{A} / \bar{P}$ and $\bar{f}=1 / 16$, Eq. (6) can be rewritten as:

$i=\frac{1}{128 g} \frac{\bar{P}}{\bar{A}^{3}} Q^{2}$

The wetted perimeter $\bar{P}$ and the water area $\bar{A}$ are expressed respectively as [8]:

$\bar{P}=\frac{B}{8}\left[4 \overline{\zeta_{n}} \sqrt{1+16 \bar{\zeta}_{n}^{2}}+\ln \left(4 \bar{\zeta}_{n}+\sqrt{1+16 \bar{\zeta}_{n}^{2}}\right)\right]$

$\bar{A}=\frac{2}{3} B^{2} \bar{\zeta}_{n}^{3}$

Inserting Eq. (8) and Eq. (9) into Eq. (7) and rearranging leads to:

$Q^{*}=\frac{\zeta_{n} / 2}{\left[4 \bar{\zeta}_{n} \sqrt{1+16 \bar{\zeta}_{n}^{2}}+\ln \left(4 \bar{\zeta}_{n}+\sqrt{1+16 \bar{\zeta}_{n}^{2}}\right)^{1 / 2}\right.}$

where $Q^{*}$ is the relative conductivity expressed as:

$Q^{*}=\frac{3 \sqrt{3}}{64 \sqrt{2 g i B^{5}}}$

All the parameters of Eq. (11) are known, which allows determining the value of the relative conductivity $Q^{*}$. What is needed is the computation of the aspect ratio $\bar{\zeta}_{n}$ using Eq.(10) for the given value of $Q^{*}$. However, as one can observe, equation (10) is implicit with respect to $\bar{\zeta}_{n}$. The computation involves iterative procedure of graphical method. One way to avoid this is to provide an approximate relationship for Eq. (10). In an axis logarithmic system, the graphical representation of Eq. (10) showed a flat curve. This suggests approximating Eq. (10) by the following handy and explicit power law:

$\overline{\zeta_{n}}=\alpha Q^{*}$

In order to obtain a better accuracy, the curve $\zeta_{n}\left(Q^{*}\right)$ expressed by Eq. (10) has been divided into four segments. Each segment was approximated by the power law given by Eq. (12). The obtained values of the parameters $\alpha$ and $\beta$ of Eq. (12) are circumstantially reported in Table 1. Also indicated is the maximum 
Table 1. Values of $\alpha$ and $\beta$ for computation of the aspect ratio $\bar{\zeta}_{n}$ by Eq. (12)

\begin{tabular}{|c|c|c|c|c|}
\hline$Q^{*}$ & $\zeta_{n}$ & $\alpha$ & $\beta$ & Maximum deviation \% \\
\hline$Q^{*} \leq 0.00261$ & $\zeta_{n} \leq 0.30$ & 1.366 & 0.255 & 0.26 \\
\hline $0.00261 \leq Q^{*} \leq 0.0182$ & $0.30 \leq \zeta_{n} \leq 0.50$ & 1.441 & 0.264 & 0.15 \\
\hline $0.0182 \leq Q^{*} \leq 0.232$ & $0.50 \leq \zeta_{n} \leq 1$ & 1.487 & 0.272 & 0.26 \\
\hline $0.232 \leq Q^{*} \leq 69.5$ & $1 \leq \zeta_{n} \leq 5$ & 1.507 & 0.282 & 0.32 \\
\hline
\end{tabular}

deviation between Eq. (10) and Eq. (12), depending on the limitations of $Q^{*}$. As it can be seen, Eq. (12) is quiet satisfactory for practical applications.

\section{NON-DIMENSIONAL CORRECTION FACTOR OF LINEAR DIMENSION}

The rough model method states that any linear dimension $L$ of a channel and the linear dimension $L$ of its rough model are related by the following equation, applicable to the whole domain of the turbulent flow:

$$
L=\psi \bar{L}
$$

where $\psi$ is a non-dimensional correction factor of linear dimension, less than unity, which is governed by the following relationship $[12,13]$ :

$\psi \cong 1.35\left[-\log \left(\frac{\varepsilon / \overline{D_{h}}}{4.75}+\frac{8.5}{\bar{R}}\right)\right]^{-2 / 5}$

where $\bar{R}$ is the Reynolds number in the rough model given by :

$\bar{R}=\frac{4 Q}{\bar{P} v}$

\section{COMPUTATION STEPS OF NORMAL DEPTH}

To compute the normal depth $y_{n}$ in a channel of parabolic cross section, the following parameters must be given: the discharge $Q$, the geometric elements $T_{m}$ and $Y_{m}$, the longitudinal slope $i$, the absolute roughness $\varepsilon$ and the kinematic viscosity $v$. All these parameters are measurable in practice. The normal depth $y_{n}$ can be computed according to the following steps:

1. Using Eq. (5), compute the parameter $B$.

2. According to Eq. (11), compute the relative conductivity $Q^{*}$.

3. Compute the aspect ratio $\overline{\zeta_{n}}$ using Eq. (12). The parameters $\alpha$ and $\beta$ are taken from Table $\mathbf{1}$, depending on the calculated value of $Q^{*}$.

4. As a result, Eq. (8) and Eq. (9) give the wetted perimeter $\bar{P}$ and the water area $\bar{A}$ respectively. This allows deducing the hydraulic diameter $\overline{D_{h}}=4 \bar{A} / \bar{P}$ and Reynolds number $\bar{R}$ by the use of Eq. (15).

5. Thus, compute the non-dimensional correction factor of linear dimension $\psi$ by applying the explicit Eq. (14).

6. Assign to the rough model the new linear dimension $\bar{B}=B / \psi$ according to Eq. (13) and derive the corresponding value of the relative conductivity $Q^{*}$ using Eq. (11).

7. Introducing this value of $Q^{*}$ into Eq. (12), we obtain the aspect ratio $\bar{\zeta}_{n}$ in the rough model equal to the aspect ratio $\zeta_{n}$ in the current channel.

8. According to Eq. (4), the required normal depth is finally as: $y_{n}=B \zeta_{n}^{2}$.

\section{EXAMPLE 1}

Compute the normal depth $y_{n}$ in the parabolic channel shown in Fig. (1), for the following data:

$Q=5 m^{3} / s, T_{m}=5 m, Y_{m}=2.5 m, i=10^{-3}, \varepsilon=10^{-3} m$, $v=10^{-6} m^{2} / s$.

1. According to Eq. (5), the linear dimension $B$ is:

$B=T_{m}^{2} / Y_{m}=5^{2} / 2.5=10 m$

2. The relative conductivity $Q^{*}$ is then:

$Q^{*}=\frac{3 \sqrt{3}}{64 \sqrt{2 g i B^{5}}}=\frac{3 \times \sqrt{3}}{64 \times \sqrt{2 \times 9.81 \times 10^{-3} \times 10^{5}}}$

$=0.00916479$

3. According to Eq. (12) and Table 1, the aspect ratio $\bar{\zeta}_{n}$ in the rough model is:

$\overline{\zeta_{n}}=\alpha Q^{\beta}=1.441 \times 0.00916479^{0.264}$

$=0.41750752$

4. The wetted perimeter $\bar{P}$ was easily calculated using Eq. (10) such that: 
$\bar{P}=\frac{B}{8}\left[4 \overline{\zeta_{n}} \sqrt{1+16 \bar{\zeta}_{n}^{2}}+\ln \left(4 \bar{\zeta}_{n}+\sqrt{1+16 \bar{\zeta}_{n}^{2}}\right)\right]$

$=5.67037211 \mathrm{~m}$

According to Eq. (9), the water area $\bar{A}$ is:

$\bar{A}=\frac{2}{3} B^{2} \bar{\zeta}_{n}^{3}=\frac{2}{3} \times 10^{2} \times 0.41750752^{3}$

$=4.85178595 m^{2}$

The hydraulic diameter $\overline{D_{h}}=4 \bar{A} / \bar{P}$ is thus: $\overline{D_{h}}=4 \bar{A} / \bar{P}=4 \times 4.85178595 / 5.67037211$

$=3.42255207 \mathrm{~m}$

Using Eq. (15), the Reynolds number $\bar{R}$ in the rough model is:

$\bar{R}=\frac{4 Q}{\bar{P} v}=\frac{4 \times 5}{5.67037211 \times 10^{-6}}=3527105.39$

5. Using Eq. (14), the non-dimensional correction factor of linear dimension $\psi$ was easily calculated as:

$\psi=1.35\left[-\log \left(\frac{\varepsilon / \overline{D_{h}}}{4.75}+\frac{8.5}{\bar{R}}\right)\right]^{-2 / 5}=0.76079518$

6. Assign to the rough model the following new value of linear dimension:

$\bar{B}=B / \psi=10 / 0.76079518=13.1441422 m$

The corresponding value of the relative conductivity $Q^{*}$ is given by Eq. (11) as:

$Q^{*}=\frac{3 \sqrt{3}}{64 \sqrt{2 g i \bar{B}^{-5}}}=\frac{3 \times \sqrt{3}}{64 \times \sqrt{2 \times 9.81 \times 10^{-3} \times 13.1441422^{5}}}$

$=0.00462692$

7. Thus, Eq. (12) and Table $\mathbf{1}$ give the aspect ratio $\zeta_{n}$ in the current channel as:

$\overline{\zeta_{n}}=\zeta_{n}=\alpha Q^{\Re}=1.441 \times 0.00462692^{0.264}$

$=0.34857883$

8. According to Eq. (4), the required normal depth is:

$y_{n}=B \zeta_{n}^{2}=10 \times 0.34857883^{2}=1.215 m$

9. This step aims to verify the validity of the calculations by determining the discharge $Q$ using Chezy's equation. The discharge so calculated should be equal to the discharge given in the problem statement. Chezy's equation expresses the discharge $Q$ as:

$Q=C A \sqrt{R_{h} i}$

$C$ is the Chezy's coefficient and $R_{h}$ is the hydraulic radius.

According to the rough model method, the coefficient $C$ is related to $\psi$ by the following formula:
$C=\frac{8 \sqrt{2 g}}{\psi^{5 / 2}}$

Hence:

$C=\frac{8 \sqrt{2 g}}{\psi^{5 / 2}}=\frac{8 \times \sqrt{2 \times 9.81}}{0.76079518^{5 / 2}}=70.1891711 \mathrm{~m}^{0.5} / \mathrm{s}$ The water area $A$ is given by Eq. (9) as:

$A=\frac{2}{3} B^{2} \zeta_{n}^{3}=\frac{2}{3} \times 10^{2} \times 0.34857883^{3}$

$=2.82365594 \mathrm{~m}^{2}$

The wetted perimeter $P$ was calculated using Eq. (10) as:

$P=\frac{B}{8}\left[4 \zeta_{n} \sqrt{1+16 \zeta_{n}^{2}}+\ln \left(4 \zeta_{n}+\sqrt{1+16 \zeta_{n}^{2}}\right)\right]$

$=4.40887352 \mathrm{~m}$

The hydraulic radius $R_{h}=A / P$ is then:

$R_{h}=A / P=2.82365594 / 4.40887352$

$=0.64044839 \mathrm{~m}$

Thus, according to Chezy, the discharge $Q$ is:

$Q=C A \sqrt{R_{h} i}$

$=70.1891711 \times 2.82365594 \times \sqrt{0.64044839 \times 10^{-3}}$

$=5.0156 \mathrm{~m}^{3} / \mathrm{s}$

The discharge so calculated and that given in the problem statement are almost equal. The deviation between both is about $0.3 \%$ only, which clearly indicates the validity of the calculations.

\section{EXAMPLE 2}

The following example shows that the rough model method is also applicable in the turbulent smooth regime.

Compute the normal depth $y_{n}$ in the parabolic channel shown in Fig. (1), for the following data:

$Q=4 \mathrm{~m}^{3} / \mathrm{s}, T_{m}=6 \mathrm{~m}, \quad Y_{m}=3 \mathrm{~m}, \quad i=10^{-3}, \quad \varepsilon \rightarrow 0$, $v=10^{-6} \mathrm{~m}^{2} / \mathrm{s}$.

1. The linear dimension $B$ is:

$B=T_{m}^{2} / Y_{m}=6^{2} / 3=12 m$

2. The relative conductivity $Q^{*}$ is then:

$$
Q^{*}=\frac{3 \sqrt{3}}{64 \sqrt{2 g i B^{5}}}=\frac{3 \times \sqrt{3}}{64 \times \sqrt{2 \times 9.81 \times 10^{-3} \times 12^{5}}}
$$

$=0.00464793$

3. According to Eq. (12) and Table 1, the aspect ratio $\bar{\zeta}_{n}$ in the rough model is:

$\overline{\zeta_{n}}=\alpha Q^{\beta}=1.441 \times 0.00464793^{0.264}$

$=0.34899599$ 
4. The wetted perimeter $\bar{P}$ was easily calculated using Eq. (10) such that:

$\bar{P}=\frac{B}{8}\left[4 \bar{\zeta} \sqrt{1+16 \bar{\zeta}_{n}^{2}}+\ln \left(4 \bar{\zeta}_{n}+\sqrt{1+16 \bar{\zeta}_{n}^{2}}\right)\right]$

$=5.29924099 m$

Applying Eq. (9), the water area $\bar{A}$ is:

$\bar{A}=\frac{2}{3} B^{2} \bar{\zeta}_{n}^{3}=\frac{2}{3} \times 12^{2} \times 0.34899599^{3}$

$=4.08067989 \mathrm{~m}^{2}$

The hydraulic diameter $\overline{D_{h}}=4 \bar{A} / \bar{P}$ is thus:

$\overline{D_{h}}=4 \bar{A} / \bar{P}=4 \times 4.08067989 / 5.29924099$

$=3.08019952 \mathrm{~m}$

Applying Eq. (15), the Reynolds number $\bar{R}$ in the rough model is:

$\bar{R}=\frac{4 Q}{\bar{P} v}=\frac{4 \times 4}{5.29924099 \times 10^{-6}}=3019300.32$

5. Using Eq. (14), the non-dimensional correction factor of linear dimension $\psi$ was easily calculated as:

$\psi=1.35\left[-\log \left(\frac{8.5}{\bar{R}}\right)\right]^{-2 / 5}=0.6801447$

6. Assign to the rough model the following new value of linear dimension:

$\bar{B}=B / \psi=12 / 0.6801447=17.6433043 m$

The corresponding value of the relative conductivity $Q^{*}$ is given by Eq. (11) as:

$Q^{*}=\frac{3 \sqrt{3}}{64 \sqrt{2 g i B^{-5}}}=\frac{3 \times \sqrt{3}}{64 \times \sqrt{2 \times 9.81 \times 10^{-3} \times 17.6433043^{5}}}$

$=0.00177322$

7. Thus, Eq. (12) and Table $\mathbf{1}$ give the aspect ratio $\zeta_{n}$ in the current channel as:

$\overline{\zeta_{n}}=\zeta_{n}=\alpha Q^{\beta}=1.366 \times 0.00177322^{0.255}$

$=0.2715721$

8. According to Eq. (4), the required normal depth is:

$y_{n}=B \zeta_{n}^{2}=12 \times 0.2715721^{2}=0.885 m$

9. After verification of the calculations according to the step 9 of example 1, the computed discharge $Q$ by applying Chezy's relationship is:

$Q=4.008 m^{3} / s$

The discharge so calculated is almost equal to the discharge given in the problem statement, confirming the validity of the calculations.

\section{CONCLUSION}

The rough model method was judiciously and successfully applied to calculate normal depth in a channel of parabolic section. The method is based on practical data, easily measurable, and does not take into account neither Chezy's coefficient nor Manning's roughness coefficient. This is one of the main advantages of the method that makes it different from current methods. The rough model method states that any linear dimension of a channel is equal to the homologous linear dimension of a referential rough model, corrected by the effect of a dimensionless factor.

The method was applied to a referential rough model whose friction coefficient has been arbitrarily chosen as a constant. The Darcy-Weisbach equation, applied to this model, has led to an implicit expression of the aspect ratio of the rough model depending on the relative conductivity. The curve of this function has been replaced by four explicit equations of power law type, remarkably accurate and very handy for the engineer use. Using a non-dimensional correction factor of linear dimension, the relationship expressing the aspect ratio in the rough model allowed deducting the aspect ratio in the current channel and therefore the required normal depth.

\section{NOTATION}

$A=$ Water area

$B=$ Linear dimension

$C=$ Chezy's coefficient

$D_{h}=$ Hydraulic diameter

$f=$ Friction factor

$g=$ Acceleration due to gravity

$i=$ Longitudinal slope of the channel

$L=$ Linear dimension

$P=$ Wetted perimeter

$Q=$ Discharge

$Q^{*}=$ Relative conductivity

$R=$ Reynolds number

$R_{h}=$ Hydraulic radius

$T_{m}=$ Top width of the channel

$Y_{m}=$ Height of the channel

$y_{n}=$ Normal depth

$\varepsilon=$ Absolute roughness

$\zeta_{n}=$ Aspect ratio equal to $\sqrt{y_{n} / B}$

$\psi=$ Non-dimensional correction factor

$v=$ kinematic viscosity

\section{CONFLICT OF INTEREST}

The authors confirm that this article content has no conflict of interest. 


\section{ACKNOWLEDGEMENT}

Decleared none.

\section{REFERENCES}

[1] V.T. Chow, Open-Channel Hydraulics, Ed., McGraw Hill: New York, 1973.

[2] R.H. French, Open Channel Hydraulics, Ed., McGraw Hill: New York, 1986.

[3] F.M. Henderson, Open channel flow, Ed., MacMillan Publishing Co.: New York, 1966.

[4] P.K. Swamee, "Normal-depth equations for irrigation canals," $J$. Irrig. Drain. Eng., ASCE, vol. 120, pp. 942-948, 1994.

[5] J. Attari, and A.M. Foroghi, "Explicit formulae for estimation of normal depth in channels," in $26^{\text {th }}$ Congr. Int. Assn. for Hydr. Res., 1995, pp. 7-12.

[6] P.K. Swamee, and P.N. Rathie, "Exact solutions for normal depth problem", J. Hydraulic Res., vol. 42, no. 5, pp. 541-547, 2004.

[7] K. Babeyan-Koopaei, "Dimensionless curves for normal-depth calculations in canal sections," J. Irrig. Drain. Eng.., ASCE, vol. 127, no. 6, pp. 386-389, 2001

[8] B. Achour and M. Khattaoui, "Computation of normal and critical depths in parabolic cross sections," Open Civil Eng. J., vol. 8, pp. 1-8, 2008.

[9] J. Liu, Z. Wang, and X. Fang, "Iterative formulas and estimation formulas for computing normal depth of horseshoe cross-section tunnel," J. Irrig. Drain. Eng., ASCE, vol. 136, no. 11, 2010.

[10] A.R. Vatankha, "Explicit solutions for critical and normal depths in trapezoidal and parabolic open channels," Ain Shams Eng. J., vol. 4, no. 1, pp. 17-23, 2013.
[11] B. Achour, "Design of pressurized vaulted rectangular conduits using the rough model method," Adv. Mater. Res., vols. 779-780, pp. 414-419, 2013.

[12] B. Achour, and A. Bedjaoui, "Discussion. Exact solutions for normal depth problem," J. Hydraul.. Res., vol. 44, no. 5, pp. 715717, 2006.

[13] B. Achour, and A. Bedjaoui, "Turbulent pipe-flow computation using the rough model method (RMM)," J. Civil. Eng. Sci., vol.1, no. 1, pp. 36-41, 2012.

[14] B. Achour and S. Sehtel, "The rough model method (rmm). application to the computation of normal depth in circular conduit," Open Civil Eng. J., vol. 8, pp. 57-63, 2014.

[15] B. Achour and M. Riabi, "Design of a pressurized trapezoidal shaped conduit using the rough model method (Part 1)," Adv. Mater. Res., vols. 945-949, pp. 892-898, 2014.

[16] B. Achour, "Computation of normal depth in trapezoidal open channel using the rough model method," Adv. Mater. Res., vols. 955-959, pp. 3231-3237, 2014.

[17] M. Riabi and B. Achour, "Design of a pressurized circular pipe with benches using the rough model method (RMM) ," Adv. Mater Res., vols. 960-961, pp. 586-591, 2014.

[18] B. Achour and A. Bedjaoui, "Design of a pressurized trapezoidal shaped conduit using the rough model method (Part 2)," Appl. Mech. Mater., vols. 580-583, pp. 1828-1841, 2014.

[19] H. Darcy, "Sur les recherches expérimentales relatives au mouvement des eaux dans les tuyaux," Comptes rendus des séances de l'Académie des Sciences, no. 38, pp. 1109-1121, 1854.

[20] C.F. Colebrook, "Turbulent flow in pipes, with particular reference to the transition between the smooth and rough pipe laws," J. Inst. Civil Eng., vol. 11, pp. 133-156, 1939. 